\title{
Longitudinal study of psychopathological, anthropometric and sociodemographic factors related to the level of Mediterranean diet adherence in a community sample of Spanish adolescents
}

\author{
Núria Voltas ${ }^{1,2}$, Victoria Arija ${ }^{2,3}$, Estefania Aparicio ${ }^{2,3}$ and Josefa Canals 1,2,* \\ 'Research Center for Behavioral Assessment (CRAMC), Department of Psychology, Universitat Rovira i Virgili, \\ Crta/ de Valls s/n, 43007 Tarragona, Spain: ${ }^{2}$ Nutrition and Mental Health Research Group (NUTRISAM), \\ Universitat Rovira i Virgili, Reus, Spain: ${ }^{3}$ Nutrition and Public Health Unit, Universitat Rovira i Virgili, Reus, Spain
}

Submitted 28 April 2015: Final revision received 27 June 2015: Accepted 26 November 2015: First published online 28 January 2016

\begin{abstract}
Objective: The Mediterranean diet (MD) pattern has important health benefits; however, it seems that Spanish school-aged children have been abandoning this healthy pattern recently. We aimed to identify psychopathological, anthropometric and sociodemographic factors that may influence the risk of low MD adherence. Design: Longitudinal study in three phases. MD adherence was assessed using the Krece Plus food questionnaire and psychopathological symptoms using the Screen for Childhood Anxiety Related Emotional Disorders, Children's Depression Inventory, Youth's Inventory-4 and Eating Disorder Inventory-2. Anthropometric data were collected in the first and third phases.

Settings: All five representative areas in Reus, Spain.

Subjects: Adolescents ( $n$ 241).

Results: Regardless of past and current BMI, socio-economic status was a protective factor for low MD adherence $(\mathrm{OR}=0.805, P=0.003)$ and a risk factor for high BMI $(\mathrm{OR}=0.718, P=0.002 ; \mathrm{OR}=0.707, P=0.001)$. Regardless of socio-economic status, depression was involved with risk of low adherence (OR=1.069, $P=0 \cdot 021)$. Girls with lower MD adherence presented significantly higher scores for eating disorders measured using the Eating Disorder Inventory-2 (low adherence, mean 18.9 (SD 13.5); high adherence, mean 8.9 (SD 9.0), $P=0.020$ ) and the Youth Inventory-4 (low adherence, mean 5.2 (SD 4.3); medium adherence, mean 3.6 (SD 3.2), $P=0.044$ ). They also presented higher depression symptoms (low adherence, mean $17 \cdot 7$ (SD 9.6); medium adherence, mean $12 \cdot 3$ (SD 7.2), $P=0.01$ ) than girls with high adherence.

Conclusions: The results highlight the influence of psychosocial factors on levels of MD adherence. These factors need to be taken into account when developing prevention and health promotion initiatives.
\end{abstract}

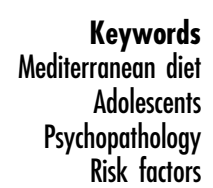

The Mediterranean diet (MD) pattern is widely known and considered a healthy diet option that involves the consumption of a wide variety of vegetables and cereals, low consumption of meat and dairy products, moderate consumption of wine during some meals, and olive oil as a source of fat. In overall terms, the MD pattern has been of great interest to many researchers because it seems to be positively associated with better health ${ }^{(1)}$. In specific terms, the MD pattern has been observed to reduce the risk of CVD, metabolic syndrome, diabetes mellitus, chronic degenerative diseases, allergies and asthma, and has even been shown to reduce the risk of mental illnesses such as depression, anxiety and binge eating disorders ${ }^{(2-9)}$. Despite this, some authors conclude that it seems Mediterranean inhabitants are replacing MD healthy eating habits by consumption of more processed foods and saturated fat, and decreasing their intakes of plant foods and MUFA ${ }^{(10,11)}$. This trend is particularly evident among children and adolescents, who presented low rates of MD adherence $^{(10,12-16)}$. Several authors have shown that about $50 \%$ of Spanish children and adolescents presented good MD adherence ${ }^{(17-19)}$, whereas in a recent study with participants from eight European countries, Tognon et al. ${ }^{(20)}$ found that Spanish school-aged girls showed the lowest prevalence of MD adherence. Furthermore, childhood and adolescence are considered critical periods for acquiring dietary and other important health habits. In this regard, several studies have observed that adherence to the 
characteristic MD pattern could be determined by a wide range of individual or social factors such as individuals' nutritional knowledge, age, gender, food pricing, information provided on television, family habits, family socio-economic status (SES), parental education, parental birthplace and type of school, among others ${ }^{(21-26)}$. Arriscado et $a l^{(17)}$ found that children who attended state schools, children from immigrant families and children from families with medium-to-low SES were less likely to follow the MD pattern. Moreover, Grosso et al. ${ }^{(27)}$ showed that adolescents living in urban areas presented less MD adherence. On the other hand, Costarelli et al. ${ }^{(28)}$ found that children and adolescents with good adherence to the MD pattern also showed physical well-being, psychological well-being, a greater feeling of having enough age-appropriate freedom and a good perception of their parents and school environment. On the basis of these results, we agree with Arriscado et al. ${ }^{(17)}$ who highlight the importance of identifying the factors that influence the level of adherence to the MD in order to be able to develop effective interventions and to reverse the unfortunate trend of young people giving up healthy eating habits related to the MD pattern ${ }^{(29)}$.

In addition to these studies, several other authors have also studied the relationship between MD adherence and anthropometric factors. There is some controversy regarding the relationship between obesity and MD adherence ${ }^{(30)}$. Several studies have reported that overweight or obese children and adolescents showed significantly lower levels of MD adherence ${ }^{(20,31,32)}$. Lydakis et $a l^{(31)}$ conducted a cross-sectional study which concluded that overweight and obese children had significantly lower MD adherence levels in comparison to children with a normal BMI. Schröder $e t a l .{ }^{(32)}$ also found the same tendency for the waist circumference variable. By contrast, Jennings et al. ${ }^{(33)}$ suggested that diet quality is independently associated with children's weight status. Following these results, Farajian et $a l .{ }^{(10)}$ conducted a cross-sectional study with children from ten regions in Greece and they too found no association between MD adherence and anthropometric factors. On the other hand, Tognon et al. ${ }^{(20)}$ conducted a follow-up study and found that the MD pattern was inversely associated with childhood obesity. In this regard, in order to clarify the relationship between MD adherence levels and BMI, more follow-up studies should be conducted with large samples of children and adolescents to study their eating and lifestyle habits and their weight and psychological characteristics. It is important to highlight that overweight and obesity among children and adolescents is an increasingly serious public health problem worldwide ${ }^{(34)}$. Furthermore, it is not only related to physical health; several studies have found a relationship between overweight and obesity and the presence of emotional disorders in adults as well as children and adolescents ${ }^{(35-37)}$, although this relationship has yet to be established unequivocally ${ }^{(38)}$.
In the light of the above, the present study aimed to describe the prevalence of MD adherence in a community sample of adolescents, to determine the adolescents' anthropometric, sociodemographic and psychopathological characteristics in relation to their level of MD adherence, and to assess the extent to which these factors contribute to or are associated with the risk of low MD adherence. Moreover, taking into account the possible relationship between emotional symptoms and BMI, we also aimed to use a mediational model to test whether low MD adherence may be a partial mediator of the association between emotional symptoms and high BMI. We hypothesized that the prevalence of MD adherence would be similar to that reported in other studies. We also hypothesized that individuals with lower MD adherence would present anthropometric alterations such as high BMI, more mental health problems such as an increased presence of emotional disorders or eating disorder symptoms, lower family SES or foreign nationality.

\section{Materials and methods}

\section{Participants and procedure}

A three-year longitudinal study was conducted among 245 subjects (147 girls and ninety-eight boys aged $12-15$ years; mean age 13.5 (SD 0.9) years). The participants were recruited from a three-phase epidemiological study of anxiety and depression disorders in the town of Reus (a Spanish town of 100000 inhabitants). The first phase took place during the 2006/2007 academic year, the second phase during the 2007/2008 academic year and the third phase during the 2009/2010 academic year.

Before beginning the study, the project was approved by the Universitat Rovira i Virgili's ethics committee for research on individuals and by the Catalan Government's Department of Education. A sample of subjects was then selected, and cluster sampling was conducted by randomly selecting a set of thirteen primary schools (seven state schools and six state-subsidized private schools) out of a total of twenty-six schools from all five representative areas of Reus (Catalonia, Spain). We then contacted the school boards, all of whom agreed to participate. After that, a letter was sent to all parents to inform them about the study and to ask for their written informed consent. We then conducted the study in three phases.

In the first phase of the study, the baseline sample was a group of 1514 subjects ( 794 girls and 720 boys; mean age $10 \cdot 2$ (SD 1.2) years). The subjects were selected from grades four (9-10 years old), five (10-11 years old) and six (11-12 years old) of primary school. Screening questionnaires for emotional disorder symptoms (the Screen for Child Anxiety Related Emotional Disorders (SCARED) and the Children's Depression Inventory (CDI)) were used to select a sample at risk of emotional disorders and a riskfree control sample. Also, in the first phase we assessed 
anthropometric and sociodemographic data. In the second phase, the sample consisted of 562 subjects (308 girls and 254 boys), of whom 405 were at risk of emotional disorders and 157 were controls. In the control group, one child without risk of emotional psychopathology was selected for every three children at risk of emotional psychopathology, matching for age, gender and type of school. All the participants of the second phase were contacted in the three-year follow-up although $43.6 \%$ dropped out. Of the 245 subjects who agreed to participate in the third phase, 241 (147 girls and ninety-four boys) completed the Krece Plus food questionnaire to determine MD adherence, and they also answered four subscales of the Eating Disorder Inventory-2 (EDI-2), five categories of the Youth's Inventory-4 (YI-4) and the Krece Plus short physical activity test. We also assessed their anthropometric and body composition data.

There were no psychopathological differences between the subjects who participated in the third phase and the subjects who dropped out in this last phase of the study. However, there were differences related to SES: participants with a low SES were associated with higher drop-out rates than medium- or high-SES participants $(P=0 \cdot 001)$.

The participants completed the questionnaires in groups of three or four. Researchers were present to instruct the children on how to answer the surveys and to answer any queries. The anthropometric and body composition measurements were made individually to ensure the participants' privacy.

\section{Instruments and measurements}

Krece Plus food questionnaire

The Krece Plus food questionnaire ${ }^{(39)}$ determines dietary quality. It consists of sixteen items, with a score of 1 or -1 for each item. The maximum possible score is 11 and the minimum is -5 . The participants were classified into three categories according to their total score on the questionnaire; their adherence to the MD was thus classified as high (total score $\geq 9$ ), medium (total score 6-8) or low (total score 55 ). This test was administered in phase 3 .

\section{Krece Plus short physical activity test}

The Krece Plus short physical activity test ${ }^{(40)}$ consists of two questions for quick screening of the physical activity/ inactivity level. Each question has six possible responses, with a score of $0-5$. The maximum score for the test is 10 and the minimum is 0 . According to the total score on the questionnaire, individuals are classified into three categories of physical activity level: good (total score of 9-10 for boys and 8-10 for girls), fair (total score of $6-8$ for boys and $5-7$ for girls) and bad (total score $\leq 5$ for boys and $\leq 4$ for girls). This test was administered in phase 3 .

\section{Anthropometry}

The anthropometric parameters evaluated in the initial and final phase were weight, height and waist circumference.
Weight was measured using the Tanita $^{\circledR}$ TBF-300 scale, which has an accuracy of $100 \mathrm{~g}$ and a maximum weight of $200 \mathrm{~kg}$. Waist circumference was measured using a flexible tape and height was measured using an inextensible tape measure, with a variation of $1 \mathrm{~mm}$ considered acceptable. Waist was measured at the midpoint between the iliac crest and the lower costal margin, without clothes. Weight and height were measured in a school room, with participants in bare feet, wearing light clothing and with no heavy objects in their pockets. BMI $\left(\mathrm{kg} / \mathrm{m}^{2}\right)$ was then calculated. We used the International Obesity Task Force cut-off points to classify participants according to their BMI as underweight, normal weight, overweight and obese ${ }^{(41,42)}$.

\section{Bioelectrical impedance}

The Tanita ${ }^{\circledR}$ TBF-300 body composition analyser was used to assess body composition. The results were expressed as body fat percentage.

\section{Screen for Childhood Anxiety and Related Emotional Disorders}

The SCARED ${ }^{(43)}$ is a forty-one-item self-report questionnaire that assesses anxiety disorder symptoms in children and adolescents aged 8-18 years. Participants were asked about the frequency of each symptom on a 3-point Likert-type scale: 0 (almost never), 1 (sometimes) and 2 (often). The Spanish version has a reliability of $\alpha=0.86$ and consists of four factors: somatic/panic, social phobia, generalized anxiety and separation anxiety ${ }^{(44)}$. A score of 25 was considered to be the cut-off point for risk of anxiety ${ }^{(45,46)}$. The SCARED was administered in all three phases.

\section{Children's Depression Inventory}

The $\mathrm{CDI}^{(47,48)}$ is a twenty-seven-item self-report inventory for assessing depression in individuals aged 7-17 years old. The children selected the sentence that best described them over the two previous weeks. The reliability of this version has been reported to be good $(\alpha=0 \cdot 81-0 \cdot 85)^{(49)}$ and was shown to be so in the present study $(\alpha=0 \cdot 83)$. A score of 17 was considered to be the cut-off point for risk of depression ${ }^{(50)}$. The CDI was administered in phases 1 and 2 .

\section{Youth's Inventory-4}

The YI- ${ }^{(51)}$ is a 120 -item self-report rating scale that evaluates the symptoms described in the Diagnostic and Statistical Manual of Mental Disorders IV for emotional and behaviour disorders in youths aged $12-18$ years. To obtain our results, we considered the following categories: attention deficit hyperactivity disorder, anxiety, depression, conduct disorder and eating disorder. In the present study we used the Spanish version of the YI- 4 , which demonstrated satisfactory internal consistency $(\alpha=0.95)$ in our sample. The YI- 4 was administered in phase 3. 
Eating Disorder Inventory-2

The EDI-2 ${ }^{(52)}$ is a ninety-one-item self-report measure of the cognitive and behavioural characteristics commonly associated with anorexia nervosa and bulimia nervosa. Responses are made on a 6-point Likert-type scale ranging from 'never' to 'always'. We used twenty-nine of the ninetyone items from the four subscales ('Drive for thinness', 'Bulimia', 'Body dissatisfaction' and 'Perfectionism'). In the present study the EDI-2's internal consistency was $\alpha=0 \cdot 80$. The EDI-2 was administered in phase 3 .

\section{Sociodemographic questionnaire}

A sociodemographic questionnaire designed by the authors for the study was used to assess the sociodemographic characteristics of the sample. The children answered questions about age, gender, place and date of birth, family type and parental occupation. This information was corroborated by their parents. SES was established using the Hollingshead index ${ }^{(53)}$, which determines an individual's social status by placing his/her occupation into one of nine categories (from unskilled work to highly skilled work) and his/her level of education into one of seven categories (from non-completed primary education to completed higher education). The status score is estimated by multiplying the occupation scale value by a weight of 5 , the education scale value by a weight of 3 and then combining the two scores. For the current study, family SES was determined by combining the data obtained from the father and the mother. The scores ranged from 0 to 66; therefore, to obtain three categories (low, medium and high) we considered scores below 22 to be low SES, those between 23 and 44 to be medium SES, and those above 44 to be high SES. This questionnaire was administered in phase 1.

\section{Statistical analyses}

Statistical analysis was performed using the statistical software package IBM SPSS Statistics Version 22.0.

We analysed descriptive data of the sociodemographic, anthropometric, MD adherence, physical activity and psychopathological variables by gender. We therefore used the $\chi^{2}$ test and Student's $t$ test depending on the types of variables compared.

The $\chi^{2}$ test was also applied to study the relationship between sociodemographic and anthropometric characteristics from the third phase and MD adherence level. We carried out ANOVA, adjusted for the Bonferroni multiple comparisons, by gender, to explore the way in which physical activity and psychopathological factors may be related to MD adherence.

Logistic regression models adjusted for age, gender, SES and the risk/control variable were applied in order to assess which variables predicted low MD adherence in our sample. The risk/control variable was created in order to neutralize the possible bias of the sample, due to a high proportion of participants being at risk of emotional disorders from the second phase. Before performing the regression models, collinearity between the variables was assessed by computing Pearson correlations between the candidate variables. We used two models. Model 1 used first-phase variables as predictors (birthplace, family type, school type, BMI, SCARED total score and CDI total score). In model 2, the predictors were the following third-phase variables: EDI-2 total score, YI-4 scores in conduct disorder, attention deficit hyperactivity disorder, anxiety and depressive symptoms, body fat percentage, BMI and the physical activity factor.

A series of regression analyses was conducted to determine if MD adherence mediates the relationship found in some studies between emotional symptoms and overweight and obesity. Gender, age and SES were included as covariables. The analysis was consistent with recommendations regarding mediational analyses in population-based research ${ }^{(54)}$.

In all the analyses, the level of statistical significance was a $P$ value $<0 \cdot 05$.

\section{Results}

Table 1 shows the descriptive data by gender for sociodemographic factors (age, SES, family type, birthplace and school type), anthropometric factors (BMI and body fat percentage), MD adherence factor, physical activity factor and psychopathological factors (SCARED, CDI, EDI-2 and YI-4). In this sample, $48.1 \%$ of adolescents presented low MD adherence, $41.9 \%$ medium MD adherence and 10.0\% presented high MD adherence. As seen in Table 1, significant differences between genders were found only for body fat percentage and psychopathological symptoms. Girls showed higher levels of body fat than boys and also obtained higher scores than boys in symptoms related to eating disorders (EDI-2 total and YI-4 scores, drive for thinness and body dissatisfaction). As for MD adherence level (see Table 2), statistically significant results were found for SES; $54 \cdot 2 \%$ of the adolescents with low SES showed low MD adherence, whereas $72.6 \%$ of those with high SES showed medium and high MD adherence. There were no significant differences in MD adherence level according to BMI although the results showed that no boy or girl with obesity presented a high level of MD adherence (see Table 3). Regarding physical activity, only boys showed statistically significant differences for MD adherence level. In relation to psychopathological factors, Table 3 shows that girls with low MD adherence presented significantly higher scores for eating disorders, and in particular for drive for thinness, anxiety and depressive symptoms, than girls with medium or high MD adherence. However, the post hoc analyses did not show statistically significant results for YI-4 anxiety symptoms or for EDI-2 drive for thinness factor scores.

Table 4 reports on the analysis of the risk of low MD adherence. In specific terms, we performed two logistic 
Table 1 Characteristics of the community sample of Spanish adolescents in terms of sociodemographic, anthropometric, MD adherence, physical activity and psychopathological factors

\begin{tabular}{|c|c|c|c|c|c|c|c|}
\hline & \multicolumn{2}{|c|}{ Total $(n$ 241) } & \multicolumn{2}{|c|}{ Boys ( $n$ 94) } & \multicolumn{2}{|c|}{ Girls ( $n$ 147) } & \multirow{2}{*}{$\begin{array}{c}P \text { value between } \\
\text { boys and girls }\end{array}$} \\
\hline & Mean & SD & Mean & SD & Mean & SD & \\
\hline Age (years) $\dagger$ & 13.5 & 0.9 & $13 \cdot 4$ & 1.0 & $13 \cdot 6$ & 0.9 & 0.098 \\
\hline Gender (\%) & - & & $38 \cdot 1$ & & 61.9 & & \\
\hline \multicolumn{8}{|l|}{ SES level (\%) } \\
\hline Low & 34.4 & & 33.7 & & 34.9 & & 0.884 \\
\hline Medium & 44.4 & & 43.5 & & $45 \cdot 0$ & & \\
\hline High & $21 \cdot 2$ & & $22 \cdot 8$ & & $20 \cdot 1$ & & \\
\hline \multicolumn{8}{|l|}{ Family type (\%) } \\
\hline Nuclear & 84.2 & & 88.0 & & 84.2 & & 0.202 \\
\hline Single-parent & $15 \cdot 8$ & & $12 \cdot 0$ & & $18 \cdot 1$ & & \\
\hline \multicolumn{8}{|l|}{ Birthplace (\%) } \\
\hline Native & $90 \cdot 5$ & & $90 \cdot 2$ & & $90 \cdot 6$ & & 0.921 \\
\hline Foreign & 9.5 & & 9.8 & & 9.4 & & \\
\hline \multicolumn{8}{|l|}{ School type (\%) } \\
\hline State school & 34.0 & & 35.9 & & 32.9 & & 0.635 \\
\hline State-subsidized private school & 66.0 & & $64 \cdot 1$ & & $67 \cdot 1$ & & \\
\hline \multicolumn{8}{|l|}{ BMI category, phase $1(\%)$} \\
\hline Underweight/normal weight & 73.3 & & 74.4 & & 72.5 & & 0.775 \\
\hline Overweight/obese & $26 \cdot 8$ & & $25 \cdot 6$ & & 27.5 & & \\
\hline \multicolumn{8}{|l|}{ BMI category, phase 3 (\%) } \\
\hline Underweight/normal weight & $85 \cdot 0$ & & 83.7 & & $85 \cdot 8$ & & 0.655 \\
\hline Overweight/obese & $15 \cdot 0$ & & $16 \cdot 3$ & & $14 \cdot 2$ & & \\
\hline Body fat percentage & $18 \cdot 7$ & 8.9 & $12 \cdot 0$ & $6 \cdot 7$ & $22 \cdot 8$ & 7.5 & 0.001 \\
\hline \multicolumn{8}{|l|}{ MD adherence (\%) } \\
\hline Low & $48 \cdot 1$ & & 43.5 & & 51.0 & & 0.524 \\
\hline Medium & 41.9 & & $45 \cdot 7$ & & 39.6 & & \\
\hline High & $10 \cdot 0$ & & $10 \cdot 9$ & & 9.4 & & \\
\hline Physical activity factor (total score) & $5 \cdot 6$ & $2 \cdot 3$ & $6 \cdot 3$ & $2 \cdot 2$ & $5 \cdot 8$ & $2 \cdot 2$ & 0.001 \\
\hline \multicolumn{8}{|l|}{ Psychopathological factors, phase 1} \\
\hline SCARED: total score & 29.6 & 11.0 & $30 \cdot 1$ & $12 \cdot 6$ & $29 \cdot 3$ & $9 \cdot 8$ & 0.606 \\
\hline CDI: total score & $11 \cdot 1$ & $7 \cdot 1$ & 11.8 & $7 \cdot 3$ & $10 \cdot 6$ & $7 \cdot 0$ & 0.210 \\
\hline \multicolumn{8}{|l|}{ Psychopathological factors, phase 3} \\
\hline EDI-2: total score & 14.8 & 11.4 & $12 \cdot 7$ & $9 \cdot 2$ & $16 \cdot 2$ & $12 \cdot 5$ & 0.012 \\
\hline EDI-2: drive for thinness factor & 4.2 & 4.9 & 3.0 & 3.4 & 4.9 & $5 \cdot 6$ & 0.001 \\
\hline EDI-2: bulimia factor & 0.6 & 1.6 & 0.5 & 1.6 & 0.6 & $1 \cdot 7$ & 0.429 \\
\hline EDI-2: body dissatisfaction factor & $6 \cdot 9$ & $6 \cdot 2$ & 5.8 & $5 \cdot 7$ & 7.7 & $6 \cdot 4$ & 0.018 \\
\hline EDI-2: perfectionism factor & 3.2 & 3.6 & 3.4 & 3.5 & 3.0 & 3.7 & 0.340 \\
\hline YI-4: anxiety symptoms & $10 \cdot 4$ & 5.8 & $9 \cdot 8$ & 5.9 & $10 \cdot 7$ & $5 \cdot 7$ & 0.269 \\
\hline YI-4: depressive symptoms & 14.7 & 8.5 & $14 \cdot 1$ & 8.2 & $15 \cdot 1$ & $8 \cdot 8$ & 0.346 \\
\hline YI-4: ADHD symptoms & $15 \cdot 8$ & 8.1 & $16 \cdot 6$ & $7 \cdot 8$ & $15 \cdot 2$ & 8.2 & 0.192 \\
\hline YI-4: conduct disorder symptoms & $6 \cdot 6$ & 4.5 & $6 \cdot 8$ & 4.6 & $6 \cdot 6$ & 4.4 & 0.671 \\
\hline YI-4: eating disorder symptoms & 3.9 & 3.7 & 3.2 & 3.3 & $4 \cdot 3$ & $3 \cdot 9$ & 0.019 \\
\hline
\end{tabular}

MD, Mediterranean diet; SES, socio-economic status; SCARED, Screen for Child Anxiety Related Disorders; CDI, Children's Depression Inventory; EDI-2, Eating Disorder Inventory-2; YI-4, Youth's Inventory-4; ADHD, attention deficit hyperactivity disorder.

$P$ values $<0.05$ are highlighted in bold font.

†Data are expressed as mean and standard deviation, or as a percentage where shown.

regression multivariate models, both of which were adjusted for age, gender, SES and the risk/control variable. Model 1 was performed to find risk factors for presenting low MD adherence and model 2 was performed to support the univariate associations found in the third phase. The EDI-2 factors and the YI-4 eating disorder symptoms were found to be collinear with the EDI-2 total score. Because of this, we introduced only the EDI-2 total score to the model as a predictor. For model 1, the results showed that high SES was a protective factor against presenting a low MD adherence level $(\mathrm{OR}=0 \cdot 805, P=0 \cdot 003)$. Likewise, the results for model 2 also showed that high SES was a protective factor $(\mathrm{OR}=0.772, P=0.001)$ and that depressive symptoms were related to the risk of presenting low MD levels $(\mathrm{OR}=1 \cdot 069, P=0 \cdot 021)$.
We performed a mediational model adjusted by gender, age and SES (Fig. 1) given the possible relationship between emotional symptoms, low SES and high BMI, and the possibility that low MD adherence is a mediator between these variables and overweight/obesity. The results revealed that the risk of low MD adherence was not a mediator between depressive symptoms and overweight/obesity, or between SES and overweight/obesity. Furthermore, on the basis of the results of model 2 we tested another mediational model that positioned depressive symptoms as a partial mediator of the association between SES and risk of low MD adherence. Although depressive symptoms were related to MD adherence, they were not a mediator variable. The results revealed that lower SES was a predictor for the risk of low MD adherence and for overweight/obesity. 
Table 2 MD adherence according to sociodemographic factors in phase 3 in the community sample of Spanish adolescents $(n 241)$

\begin{tabular}{|c|c|c|c|c|c|c|c|}
\hline \multirow[b]{3}{*}{ Sociodemographic factor } & \multicolumn{6}{|c|}{ Adherence to MD } & \multirow[b]{3}{*}{$P$} \\
\hline & \multicolumn{2}{|c|}{ Low $(n 116)$} & \multicolumn{2}{|c|}{ Medium ( $n$ 101) } & \multicolumn{2}{|c|}{ High ( $n$ 24) } & \\
\hline & $n$ & $\%$ & $n$ & $\%$ & $n$ & $\%$ & \\
\hline \multicolumn{8}{|l|}{ Gender } \\
\hline Boys & 41 & 43.5 & 43 & $45 \cdot 7$ & 10 & 10.9 & \multirow[t]{2}{*}{0.524} \\
\hline Girls & 75 & $51 \cdot 0$ & 58 & 39.6 & 14 & 9.4 & \\
\hline \multicolumn{8}{|l|}{ Age group } \\
\hline $9-13$ years & 59 & $47 \cdot 2$ & 54 & $43 \cdot 2$ & 12 & $9 \cdot 6$ & \multirow[t]{2}{*}{0.912} \\
\hline $14-15$ years & 57 & $49 \cdot 1$ & 47 & 40.5 & 12 & $10 \cdot 3$ & \\
\hline \multicolumn{8}{|l|}{ Birthplace } \\
\hline Native & 100 & 45.9 & 95 & 43.6 & 23 & $10 \cdot 6$ & \multirow[t]{2}{*}{0.093} \\
\hline Foreign & 16 & 69.6 & 6 & $26 \cdot 1$ & 1 & 4.3 & \\
\hline \multicolumn{8}{|l|}{ Family type } \\
\hline Single-parent & 22 & 57.9 & 13 & 34.2 & 3 & 7.9 & \multirow[t]{2}{*}{0.422} \\
\hline Nuclear & 94 & $46 \cdot 3$ & 88 & 43.1 & 21 & $10 \cdot 3$ & \\
\hline \multicolumn{8}{|l|}{ School type } \\
\hline State schools & 40 & $48 \cdot 8$ & 37 & $45 \cdot 1$ & 5 & $6 \cdot 1$ & \multirow[t]{3}{*}{0.337} \\
\hline State-subsidized private & 76 & $47 \cdot 8$ & 64 & $40 \cdot 3$ & 19 & 11.9 & \\
\hline \multicolumn{7}{|l|}{ SES } & \\
\hline Low & 45 & $54 \cdot 2$ & 30 & $36 \cdot 1$ & 8 & $9 \cdot 6$ & \multirow{3}{*}{0.022} \\
\hline Medium & 57 & 53.3 & 40 & $37 \cdot 4$ & 10 & $9 \cdot 3$ & \\
\hline High & 14 & 27.5 & 31 & $60 \cdot 8$ & 6 & 11.8 & \\
\hline
\end{tabular}

MD, Mediterranean diet; SES, socio-economic status.

$P$ values $<0.05$ are highlighted in bold font.

Table $3 \mathrm{MD}$ adherence in relation to anthropometric, physical activity and psychopathological factors in phase 3 in the community sample of Spanish adolescents ( $n$ 241)

\begin{tabular}{|c|c|c|c|c|c|c|c|c|c|c|c|c|c|c|c|c|}
\hline & \multicolumn{16}{|c|}{ Adherence to MD } \\
\hline & \multicolumn{8}{|c|}{ Boys } & \multicolumn{8}{|c|}{ Girls } \\
\hline & \multicolumn{2}{|c|}{ Low } & \multicolumn{2}{|c|}{ Medium } & \multicolumn{2}{|c|}{ High } & \multirow[b]{2}{*}{$P$} & \multirow[b]{2}{*}{$P$} & \multicolumn{2}{|c|}{ Low } & \multicolumn{2}{|c|}{ Medium } & \multicolumn{2}{|c|}{ High } & \multirow[b]{2}{*}{$P$} & \multirow[b]{2}{*}{$P$} \\
\hline & $n$ & $\%$ & $n$ & $\%$ & $n$ & $\%$ & & & $n$ & $\%$ & $n$ & $\%$ & $n$ & $\%$ & & \\
\hline \multirow{3}{*}{$\begin{array}{l}\text { Anthropometric factor } \\
\text { BMl category, phase } 3 \\
\text { Underweight/normal weight } \\
\text { Overweight/obese }\end{array}$} & & & & & & & & & & & & & & & & \\
\hline & $\begin{array}{c}33 \\
7\end{array}$ & $\begin{array}{l}42 \cdot 9 \\
46 \cdot 7\end{array}$ & $\begin{array}{c}35 \\
7\end{array}$ & $\begin{array}{l}45 \cdot 5 \\
46 \cdot 7\end{array}$ & $\begin{array}{l}9 \\
1\end{array}$ & $\begin{array}{r}11.7 \\
6.7\end{array}$ & 0.845 & & $\begin{array}{c}67 \\
9\end{array}$ & $\begin{array}{l}52 \cdot 8 \\
42 \cdot 9\end{array}$ & $\begin{array}{l}49 \\
10\end{array}$ & $\begin{array}{l}38 \cdot 6 \\
47 \cdot 6\end{array}$ & $\begin{array}{c}11 \\
2\end{array}$ & $\begin{array}{l}8.7 \\
9.5\end{array}$ & 0.695 & \\
\hline & Mean & SD & Mean & SD & Mean & SD & $P$ & $P$ & Mean & SD & Mean & SD & Mean & $\mathrm{SD}$ & $P$ & $P$ \\
\hline $\begin{array}{l}\text { Physical activity factor } \\
\text { Psychopathological factors }\end{array}$ & $6 \cdot 5^{a}$ & $2 \cdot 1$ & $5 \cdot 8^{b}$ & $2 \cdot 3$ & $8.0^{c}$ & 0.9 & 0.010 & $0.010^{\mathrm{bc}}$ & $5 \cdot 0$ & $2 \cdot 1$ & $5 \cdot 3$ & $2 \cdot 1$ & 5.5 & 2.9 & 0.613 & \\
\hline EDI-2: total score & $12 \cdot 6$ & 9.8 & $13 \cdot 1$ & 9.4 & 11.0 & 6.4 & 0.805 & & $18.9^{a}$ & 13.5 & $14 \cdot 3^{b}$ & 10.7 & $8.9^{c}$ & 9.0 & 0.008 & $0.020^{\mathrm{ac}}$ \\
\hline EDI-2: drive for thinness factor & $\begin{array}{r}2.6 \\
0.8\end{array}$ & $\begin{array}{ll}3.0 \\
2.5\end{array}$ & 3.7 & 4.0 & 1.8 & 1.5 & 0.180 & & $\begin{array}{r}5.9 \\
0.8\end{array}$ & $\begin{array}{r}6 \cdot 2 \\
1.8\end{array}$ & $4 \cdot 1$ & $\begin{array}{r}4.7 \\
1.7\end{array}$ & $2 \cdot 4$ & $\begin{array}{l}3.9 \\
0.3\end{array}$ & $\mathbf{0 . 0 3 8}$ & \\
\hline $\begin{array}{l}\text { EDI-2: bulimia factor } \\
\text { EDI-2: body dissatisfaction } \\
\text { factor }\end{array}$ & $\begin{array}{l}0.8 \\
5 \cdot 9\end{array}$ & $\begin{array}{l}2 \cdot 2 \\
5 \cdot 2\end{array}$ & $\begin{array}{l}0.2 \\
5 \cdot 9\end{array}$ & $\begin{array}{l}0.7 \\
6 \cdot 3\end{array}$ & $\begin{array}{l}0.3 \\
4.8\end{array}$ & $\begin{array}{l}0.5 \\
5 \cdot 0\end{array}$ & $\begin{array}{l}0.281 \\
0.857\end{array}$ & & $\begin{array}{l}0.8 \\
8.7\end{array}$ & $\begin{array}{l}1 \cdot 8 \\
6 \cdot 8\end{array}$ & $\begin{array}{l}0.5 \\
7.1\end{array}$ & $\begin{array}{l}1 \cdot 7 \\
5 \cdot 8\end{array}$ & $\begin{array}{l}0.1 \\
4.7\end{array}$ & $\begin{array}{l}0.3 \\
5 \cdot 8\end{array}$ & $\begin{array}{l}0.310 \\
0.076\end{array}$ & \\
\hline EDI-2: perfectionism factor & 3.4 & 3.8 & 3.4 & 3.2 & $4 \cdot 1$ & $3 \cdot 1$ & 0.816 & & 3.5 & 4.3 & 2.6 & 2.9 & 1.8 & $2 \cdot 1$ & 0.157 & \\
\hline YI-4: anxiety symptoms & $9 \cdot 2$ & $6 \cdot 2$ & $10 \cdot 9$ & $5 \cdot 6$ & 7.9 & $5 \cdot 0$ & 0.225 & & 11.8 & $6 \cdot 4$ & $9 \cdot 6$ & 4.8 & 8.7 & 3.7 & 0.036 & \\
\hline YI-4: depressive symptoms & 14.5 & 9.3 & 14.0 & $7 \cdot 2$ & $12 \cdot 6$ & 7.6 & 0.807 & & $17 \cdot 7^{\mathrm{a}}$ & $9 \cdot 6$ & $12 \cdot 3^{\mathrm{b}}$ & $7 \cdot 2$ & $13 \cdot 0^{\mathrm{C}}$ & $5 \cdot 8$ & 0.001 & $0.001^{\mathrm{ab}}$ \\
\hline YI-4: ADHD symptoms & $35 \cdot 3$ & $17 \cdot 2$ & 31.7 & 13.7 & 31.6 & $16 \cdot 8$ & 0.560 & & 32.4 & $18 \cdot 3$ & 28.9 & $15 \cdot 0$ & $26 \cdot 14$ & 9.7 & 0.290 & \\
\hline $\begin{array}{l}\text { YI-4: conduct disorder } \\
\text { symptoms }\end{array}$ & $7 \cdot 6$ & $6 \cdot 0$ & $6 \cdot 2$ & $3 \cdot 1$ & $6 \cdot 1$ & $3 \cdot 2$ & 0.331 & & $7 \cdot 1$ & 4.9 & $6 \cdot 1$ & 3.7 & $5 \cdot 6$ & $3 \cdot 2$ & 0.317 & \\
\hline YI-4: eating disorder symptoms & 3.2 & 3.4 & 3.0 & 3.4 & 3.7 & 3.2 & 0.844 & & $5 \cdot 2^{\mathrm{a}}$ & $4 \cdot 3$ & $3.6^{\mathrm{b}}$ & $3 \cdot 2$ & $2 \cdot 9^{\mathrm{c}}$ & 3.0 & 0.018 & $0.044^{\mathrm{ab}}$ \\
\hline
\end{tabular}

MD, Mediterranean diet; EDI-2, Eating Disorder Inventory-2; YI-4, Youth's Inventory-4; ADHD, attention deficit hyperactivity disorder.

$P$ values $<0.05$ are highlighted in bold font.

$\mathrm{bc}, \mathrm{ac}, \mathrm{ab}$ Mean values that were significantly different by Bonferroni analysis.

\section{Discussion}

In the present study, only $10.0 \%$ of our adolescent participants showed high levels of MD adherence as determined by the Krece Plus food questionnaire. Likewise, a study performed by De Rufino et al. ${ }^{(55)}$ with a sample of adolescents aged between 10 and 17 years also found a low nutritional risk of $15 \%$. Both these sets of results are low, 
Table 4 Logistic regression models to predict risk of low MD adherence according to sociodemographic, anthropometric, physical activity and psychopathological factors in the community sample of Spanish adolescents $(n 241)$

\begin{tabular}{|c|c|c|c|}
\hline & \multicolumn{3}{|c|}{ Risk of low MD adherence } \\
\hline & OR & $95 \% \mathrm{Cl}$ & $P$ \\
\hline \multicolumn{4}{|l|}{ Model 1: phase 1 variables } \\
\hline Gender & 0.80 & $0.45,1.42$ & 0.452 \\
\hline Age & 0.91 & $0.66,1.25$ & 0.549 \\
\hline Birthplace & $2 \cdot 14$ & $0.80,5.72$ & 0.130 \\
\hline Family type & 1.41 & $0.66,3.05$ & 0.377 \\
\hline School type & 0.89 & $0.49,1.61$ & 0.698 \\
\hline SES & 0.805 & $0.698,0.932$ & 0.003 \\
\hline Risk/control & 1.707 & $0.758,3.848$ & 0.197 \\
\hline BMI category, phase 1 & 0.971 & $0.890,1.059$ & 0.500 \\
\hline Total SCARED & 1.006 & $0.972,1.041$ & 0.741 \\
\hline Total CDI & 0.973 & $0.926,1.022$ & 0.268 \\
\hline \multicolumn{4}{|c|}{$R^{2}$ Nagelkerke $\times 100=10 \cdot 8, \chi_{10228}^{2}=19.148, P=0.038$} \\
\hline \multicolumn{4}{|l|}{ Model 2: phase 3 variables } \\
\hline Gender & 0.497 & $0 \cdot 200,1 \cdot 235$ & 0.132 \\
\hline Age & 0.884 & $0.653,1.197$ & 0.426 \\
\hline SĚS & 0.772 & $0.663,0.899$ & 0.001 \\
\hline Total EDI-2 & 1.018 & $0.985,1.053$ & 0.291 \\
\hline YI-4 conduct disorder symptoms & 1.034 & $0.949,1.127$ & 0.442 \\
\hline YI-4 ADHD symptoms & 0.999 & $0.973,1.025$ & 0.921 \\
\hline YI-4 anxiety symptoms & 0.934 & $0.867,1.006$ & 0.072 \\
\hline YI-4 depressive symptoms & 1.069 & $1.010,1.132$ & 0.021 \\
\hline Body fat percentage & 0.970 & $0.903,1.043$ & 0.411 \\
\hline BMI category, phase 3 & 1.004 & $0.853,1.182$ & 0.961 \\
\hline Physical activity factor & 1.054 & $0.918,1.211$ & 0.454 \\
\hline Risk/control & 1.536 & $0.812,2.903$ & 0.187 \\
\hline \multicolumn{4}{|c|}{$R^{2}$ Nagelkerke $\times 100=17.3, \chi_{12231}^{2}=32.072, P=0.001$} \\
\hline
\end{tabular}

MD, Mediterranean diet; SES, socio-economic status; SCARED, Screen for Child Anxiety Related Emotional Disorders; CDI, Children's Depression Inventory; EDI-2, Eating Disorder Inventory-2; YI-4, Youth's Inventory-4; ADHD, attention deficit hyperactivity disorder.

Models are adjusted for gender, age, SES and risk/control; $P$ values $<0.05$ are highlighted in bold font.

Scores were assigned to quantitative variables as follows (no score for categorical variables): age; SES; 1st phase BMI; total SCARED; CDI; total EDI; YI-4 conduct disorder symptoms; YI-4 anxiety symptoms; YI-4 depressive symptoms; body fat; 3rd phase BMI; physical activity factor; gender (1=boy; 2 = girl); birthplace $(0=$ foreign; $1=$ native); family type $(0=$ single-parent; $1=$ nuclear); school type $(1=$ state school; $2=$ state-subsidized private school); risk/control $(1=$ risk; 2 = control).

particularly if they are compared with the levels of MD adherence found in the study by Serra-Majem et al. ${ }^{(29)}$ or the results of a recent study conducted with Spanish school-aged children $^{(17)}$, although those studies used the KIDMED questionnaire, which has different cut-off points. The results from the present study may be lower because it studied a population with a considerable number of individuals at risk of emotional disorders. In order to prevent this factor from distorting the results, we controlled for this variable in the multivariate models. Almost $50 \%$ of the participants presented low MD adherence and, although we found no relationship with anthropometric data, this is of concern given the high rates of overweight and obesity affecting the Spanish population $^{(56,57)}$ and the health benefits of the $\mathrm{MD}^{(1)}$.

The results showed that $54.2 \%$ of adolescents with low SES reported low MD adherence and $72.6 \%$ of those with high SES reported medium to high MD adherence. We also found that high SES was a protective factor against presenting low MD adherence and overweight/obesity. Therefore, as in other studies, it seems that families with higher SES have better MD patterns than those with lower $\operatorname{SES}^{(17,27,58)}$. This result is open to multiple interpretations. First, families with high incomes usually have high levels of education which may increase their awareness of healthy dietary habits and, therefore, may make them more likely to follow MD patterns. Second, their better economic opportunities may lead them to consume higher-quality and healthier products. Regarding the other sociodemographic factors, our results did not show any statistically significant differences for MD adherence according to school type or nationality, as were found by Arriscado et al. ${ }^{(17)}$. This may be because our sample has a small number of foreigners.

We observed no significant relationships between the past and current BMI factor and MD adherence. Despite this, our results showed that no boy or girl with obesity presented high MD adherence. However, although our results are not statistically significant, it is worth pointing out that all participants with a BMI indicative of obesity followed diet patterns that deviated from the MD and that, as shown by Grosso et $a l .{ }^{(27)}$, these participants probably ate unhealthy food which affected their BMI. This may be supported by the relationship found between high BMI and low SES, and the interpretations mentioned above. Our findings also showed that risk factors for low MD adherence were independent of anthropometric data such 
(a)

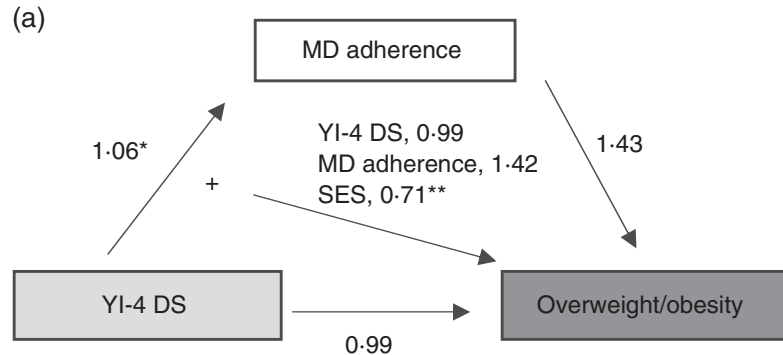

(b)

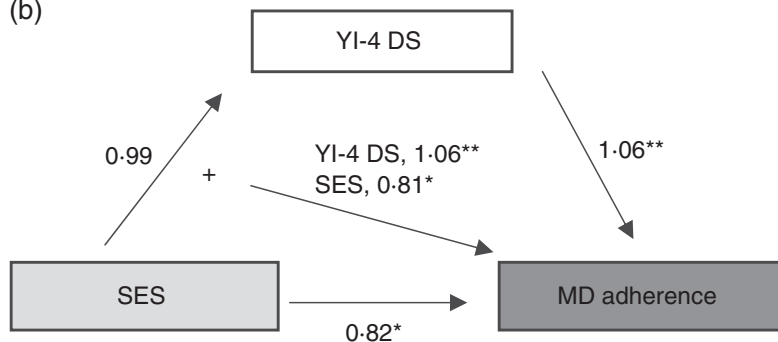

(c)

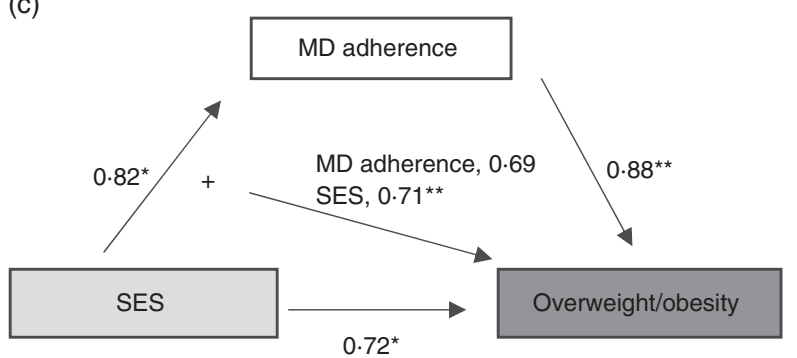

Fig. 1 Results of regression analyses conducted to determine if MD adherence mediates the relationship between emotional symptoms and overweight/obesity in a community sample of Spanish adolescents ( $n$ 241). All analyses of mediation controlled for age, gender and/or SES. Independent variables are shown in light grey boxes, mediator variables in white boxes and dependent variables in dark grey boxes; ${ }^{*} P<0.05$, ${ }^{\star *} P<0.01$ (MD, Mediterranean diet; SES, socio-economic status; YI-4 DS, Youth's Inventory-4 depressive symptoms)

as past and current BMI or body fat. In this regard, Rossi et $a l .{ }^{(59)}$ showed that, in adults, being classified as underweight, normal weight, overweight and obese had no significant effect on MD adherence. In relation to the controversy surrounding the relationship between anthropometric data and MD adherence during childhood and adolescence, the present results are consistent with other studies which have found no evidence of such a relationship ${ }^{(10,33)}$. However, although no relationship between MD adherence and anthropometric factors was found, we cannot rule out this relationship being observed in the future. We believe that not following the MD pattern may lead to undesirable life habits or habits which may lead to negative anthropometric effects in the long term, such as higher BMI ${ }^{(60,61)}$. Furthermore, although physical activity was not related to MD adherence in the multivariate analyses, we found that boys with high MD adherence presented high levels of physical activity. Our results also showed that girls presented lower physical activity and higher levels of body fat than boys, coinciding with data in recent studies which concluded that girls are less active and more sedentary than boys, even after taking into account physiological differences in body fat between the genders ${ }^{(62,63)}$. However, although healthy dietary habits are known to be mostly associated with physical activity ${ }^{(62)}$, the present study showed no significant differences between boys and girls in terms of MD adherence level.

Several authors have suggested that there is a significant association between unhealthy dietary patterns and poor mental health in children, adolescents and adults, and this is especially true for depression and anxiety ${ }^{(7,64-66)}$. In a recent large national longitudinal survey of Canadians aged 18 years and older, Kingsbury et $a l^{(67)}$ suggested that relationships between healthy intake (fruit and vegetables) and depression are complex. Behaviours such as smoking and poor physical activity may have a more important impact on depression than fruit and vegetable intake. From a biological point of view, there are several potential pathways by which diet quality may be related to mental health ${ }^{(68)}$. In specific terms, isolated nutrients or foods in the MD, such as B-vitamins, folate and $n-3$ fatty acids, are known to have preventive effects on depression in adults. The MD is also related to reductions in vascular, inflammatory and metabolic processes in patients with depression. For example, folate is required to synthesize methionine, which is a precursor of $S$-adenosylmethionine, and it acts in methylation reactions such as those involving neurotransmitters with antidepressant characteristics ${ }^{(7,69-73)}$. When we studied the psychopathological characteristics of the sample in relation to their MD adherence level, the results showed that girls with low MD adherence reported high mean scores of depression and eating disorder symptoms. Regardless of gender, in data from multivariate analyses Fulkerson et al. ${ }^{(74)}$ found that poor dietary quality was cross-sectionally associated with depression symptoms. As in Jacka et al. ${ }^{(75)}$, our results did not show that baseline depression was a predictive factor for diet quality during follow-up. The relationship found leads us to believe that girls with depressive symptoms are more likely to consume comfort foods, such as sweet snack foods, rather than healthy MD foods as a way to reduce feelings of sadness ${ }^{(76,77)}$. In fact, this behaviour is also observed in eating disorders characterized by binge eating episodes. Consequently, several of the variables studied may be bidirectionally related and therefore we performed three mediational models to explain whether MD adherence or depressive symptoms were mediators of overweight/obesity and diet quality respectively. In this regard, despite depressive symptoms being related to MD adherence and SES, this variable was not a mediator for high BMI. Low SES was also a predictor of risk for overweight and obesity. These results suggest that children's diet patterns and BMI depend on their family's socio-economic and educational level and their parent's 
health awareness, as argued by Sotos-Prieto et al. ${ }^{(78)}$. In a large percentage of cases, when a child comes from a low socio-economic environment his/her diet quality is worse, and this was not mediated by depressive symptoms. Likewise, the relationship between low SES and overweight/obesity may be explained by other factors as well as diet, such as physical activity.

The current study has both strengths and limitations. In terms of its strengths, it presents a prospective study of data found in a community sample of adolescents, although unfortunately we have no data on the participants' MD adherence in the first phase that would allow us examine whether the MD has a potential role in preventing mental health problems in children. Furthermore, a larger sample would be preferable. Also, there were differences in the SES factor between adolescents who participated in the third phase and adolescents who decided to not participate, but this was taken into account by using the SES variable as a control variable during the analyses. Moreover, we think that the Krece Plus short physical activity test may be too simple to evaluate physical activity habits and that it would have been better to use another tool.

As we had hypothesized, we observed significant differences in MD adherence level when SES was taken into account. However, none of the other variables such as age, nationality, family type or school type were found to be related to or influenced by $\mathrm{MD}$ adherence level. As for the findings on depressive symptoms, and especially in the case of girls, we conclude that this factor and low SES are related to low diet quality and we believe that these two factors should be taken into consideration in programmes designed to prevent obesity and promote dietary health. In short, the results highlighted the influence of psychosocial factors on MD adherence level. In our opinion, abandoning the MD pattern may have long-term negative anthropometric consequences. Strategies are needed to help children and adolescents obtain more knowledge about healthy nutritional habits, the benefits of the MD pattern and other healthy dietary patterns, and other healthy habits such as regular physical activity. These kinds of initiative could be carried out in schools and could involve families as a whole.

\section{Acknowledgements}

Acknowledgements: The authors are grateful to all the schools and children that participated in the study. Financial support: This research was supported by a grant from the 'Fondo de Investigaciones Sanitarias' (PI07/0839) of the Instituto de Salud Carlos III of the Spanish Ministry of Health, Social Services and Equality. The funder had no role in the design, analysis or writing of this article. Conflict of interest: None. Authorship: The authors contributed as follows. N.V., psychological assessment during data collection, statistical analysis, author of the draft manuscript; V.A., statistical assessment and discussion of nutritional data, reviewed and approved the final version of the manuscript; E.A., nutritional assessment during data collection; J.C., managed and designed the study, psychological assessment during the first phase, collaborated in writing the manuscript and approved the final version. Ethics of human subject participation: The project was approved by the Universitat Rovira i Virgili's ethics committee for research on individuals and by the Catalan Government's Department of Education. All parents were informed about the study and gave written informed consent for their child's participation.

\section{References}

1. Sofi F, Macchi C, Abbate R et al. (2014) Mediterranean diet and health status: an updated meta-analysis and a proposal for a literature-based adherence score. Public Health Nutr 17, 2769-2782.

2. Bertoli S, Spadafranca A, Bes-Rastrollo M et al. (2015) Adherence to the Mediterranean diet is inversely related to binge eating disorder in patients seeking a weight loss program. Clin Nutr 34, 107-114.

3. Chatzi L, Apostolaki G, Bibakis I et al. (2007) Protective effect of fruits, vegetables and the Mediterranean diet on asthma and allergies among children in Crete. Thorax 62, 677-683.

4. Chiva-Blanch G, Badimon L \& Estruch R (2014) Latest evidence of the effects of the Mediterranean diet in prevention of cardiovascular disease. Curr Atheroscler Rep 16, 446.

5. Domínguez LJ, Bes-Rastrollo M, De la Fuente-Arrillaga C et al. (2013) Similar prediction of total mortality, diabetes incidence and cardiovascular events using relative- and absolute-component Mediterranean diet score: the SUN cohort. Nutr Metab Cardiovas Dis 23, 451-458.

6. Kastorini CM, Milionis HJ, Esposito K et al. (2011) The effect of Mediterranean diet on metabolic syndrome and its components: a meta-analysis of 50 studies and 534,906 individuals. J Am Coll Cardiol 57, 1299-1313.

7. Sánchez-Villegas A, Delgado-Rodríguez M, Alonso A et al. (2009) Association of the Mediterranean dietary pattern with the incidence of depression: the Seguimiento Universidad de Navarra/University of Navarra follow-up (SUN) cohort. Arch Gen Psychiatry 66, 1090-1098.

8. Salas-Salvadó J, Bulló M, Babio N et al. (2011) Reduction in the incidence of type 2 diabetes with the Mediterranean diet: results of the PREDIMED-Reus nutrition intervention randomized trial. Diabetes Care 34, 14-19.

9. Tangney CC, Kwasny MJ, Li H et al. (2011) Adherence to a Mediterranean-type dietary pattern and cognitive decline in a community population. Am J Clin Nutr $\mathbf{9 3}$, 601-607.

10. Farajian P, Risvas G, Karasouli K et al. (2011) Very high childhood obesity prevalence and low adherence rates to the Mediterranean diet in Greek children: the GRECO study. Atherosclerosis 217, 525-530.

11. Naska A \& Trichopoulou A (2014) Back to the future: the Mediterranean diet paradigm. Nutr Metab Cardiovasc Dis 24, 216-219.

12. Antonogeorgos G, Panagiotakos DB, Grigoropoulou D et al. (2013) The mediating effect of parents' educational status on the association between adherence to the Mediterranean diet and childhood obesity: the PANACEA study. Int J Public Health 58, 401-408.

13. Bibiloni M, Martínez E, Llull R et al. (2012) Western and Mediterranean dietary patterns among Balearic Islands' adolescents: socio-economic and lifestyle determinants. Public Health Nutr 15, 683-692. 
14. Hebestreit A \& Ahrens W (2010) Relationship between dietary behaviours and obesity in European children. Int $J$ Pediatr Obes 5, 45-47.

15. Kontogianni MD, Vidra N, Farmaki AE et al. (2008) Adherence rates to the Mediterranean diet are low in a representative sample of Greek children and adolescents. J Nutr 13, 1951-1956.

16. Santomauro F, Lorini C, Tanini T et al. (2014) Adherence to Mediterranean diet in a sample of Tuscan adolescents. Nutrition 30, 1379-1383.

17. Arriscado D, Muros JJ, Zabala M et al. (2014) Factors associated with low adherence to a Mediterranean diet in healthy children in northern Spain. Appetite 80, 28-34.

18. Mariscal-Arcas M, Rivas A, Velasco J et al. (2009) Evaluation of the Mediterranean Diet Quality Index (KIDMED) in children and adolescents in Southern Spain. Public Health Nutr 12, 1408-1412.

19. Martínez E, Llull R, Bibiloni M et al. (2010) Adherence to the Mediterranean dietary pattern among Balearic Islands adolescents. Br J Nutr 103, 1657-1664.

20. Tognon G, Hebestreit A, Lanfer A et al. (2014) Mediterranean diet, overweight and body composition in children from eight European countries: cross-sectional and prospective results from the IDEFICS study. Nutr Metab Cardiovas Dis 24, 205-213.

21. Cutler GJ, Flood A, Hannan P et al. (2011) Multiple sociodemographic and socioenvironmental characteristics are correlated with major patterns of dietary intake in adolescents. J Am Diet Assoc 111, 230-240.

22. Hammons AJ \& Fiese BH (2011) Is frequency of shared family meals related to the nutritional health of children and adolescents? Pediatrics 127, e1565-e1574.

23. Taylor JP, Evers S \& McKenna M (2005) Determinants of healthy eating in children and youth. Can J Public Health 96, Suppl. 3, S20-S26.

24. Papadaki S \& Mavrikaki E (2015) Greek adolescents and the Mediterranean diet: factors affecting quality and adherence. Nutrition 31, 345-349.

25. Colić-Barić I, Kajfež R, Šatalić Z et al. (2004) Comparison of dietary habits in the urban and rural Croatian schoolchildren. Eur J Nutr 43, 169-174.

26. Lazarou C \& Kalavana T (2009) Urbanization influences dietary habits of Cypriot children: the CYKIDS study. Int J Public Health 54, 69-77.

27. Grosso G, Marventano S, Giorgianni G et al. (2014) Mediterranean diet adherence rates in Sicily, southern Italy. Public Health Nutr 17, 2001-2009.

28. Costarelli V, Koretsi E \& Georgitsogianni E (2013) Healthrelated quality of life of Greek adolescents: the role of the Mediterranean diet. Qual Life Res 22, 951-956.

29. Serra-Majem Ll Ribas L, Ngo J et al. (2004) Food, youth and the Mediterranean diet in Spain. Development of KIDMED, Mediterranean Diet Quality Index in children and adolescents. Public Health Nutr 7, 931-935.

30. Buckland G, Bach A \& Serra-Majem L (2008) Obesity and the Mediterranean diet: a systematic review of observational and intervention studies. Obes Rev $9,582-593$.

31. Lydakis C, Stefanaki E, Stefanaki S et al. (2012) Correlation of blood pressure, obesity, and adherence to the Mediterranean diet with indices of arterial stiffness in children. Eur J Pediatr 171, 1373-1382.

32. Schröder H, Fitó M, Estruch R et al. (2011) A short screener is valid for assessing Mediterranean diet adherence among older Spanish men and women. J Nutr 141, $1140-1145$

33. Jennings A, Welch A, Van Sluijs EM et al. (2011) Diet quality is independently associated with weight status in children aged 9-10 years. J Nutr 141, 453-459.

34. Han JC, Lawlor DA \& Kimm SY (2010) Childhood obesity. Lancet 375, 1737-1748.
35. Aparicio E, Canals J, Voltas N et al. (2013) Emotional psychopathology and increased adiposity: follow-up study in adolescents. J Adolesc 36, 319-330.

36. Flórez KR, Dubowitz T, Ghosh-Dastidar MB et al. (2015) Associations between depressive symptomatology, diet, and body mass index among participants in the supplemental nutrition assistance program. J Acad Nutr Diet 115, 1102-1108.

37. Goodman E \& Whitaker RC (2002) A prospective study of the role of depression in the development and persistence of adolescent obesity. Pediatrics 110, 497-504.

38. Benson LP, Williams RJ \& Novick MB (2013) Pediatric obesity and depression a cross-sectional analysis of absolute BMI as it relates to children's depression index scores in obese 7- to 17-year-old children. Clin Pediatr (Phila) 52, 24-29.

39. Serra-Majem Ll, Aranceta-Bartrina J, Ribas-Barba L et al. (2003) Crecimiento y desarrollo: dimensión alimentaria y nutricional. El cribado del riesgo nutricional en pediatría. Validación del test rápido, Krece Plus y resultados en la población española. In Crecimiento y desarrollo. Estudio Enkid. Krece Plus, vol. 4, pp. 45-55 [L Serra-Majem and J Aranceta-Bartrina, editors]. Barcelona: Masson.

40. Román-Viñas B, Serra-Majem L, Ribas-Barba L et al. (2003) Crecicimiento y desarrollo: actividad física. Estimación del nivel de actividad física mediante el Test Corto Krece Plus. Resultados en la población española. In Crecimiento y desarrollo. Estudio Enkid. Krece Plus, vol. 4, pp. 57-74 [L Serra-Majem and J Aranceta-Bartrina, editors]. Barcelona: Masson.

41. Cole TJ, Bellizzi MC, Flegal KM et al. (2000) Establishing a standard definition for child overweight and obesity worldwide: international survey. BMJ 320, 1240-1243.

42. Cole TJ, Flegal KM, Nicholls D et al. (2007) Body mass index cut offs to define thinness in children and adolescents: international survey. BMJ 335, 194.

43. Birmaher B, Khetarpal S, Brent D et al. (1997) The Screen for Child Anxiety Related Emotional Disorders (SCARED): scale construction and psychometric characteristics. J Am Acad Child Adolesc Psychiatry 36, 545-553.

44. Vigil-Colet A, Canals J, Cosi S et al. (2009) The factorial structure of the 41-item version of the Screen for Child Anxiety Related Emotional Disorders (SCARED) in a Spanish population of 8 to 12 years old. Int J Clin Health Psychol 9, 313-327.

45. Birmaher B, Brent DA, Chiappetta L et al. (1999) Psychometric properties of the Screen for Child Anxiety Related Emotional Disorders (SCARED): a replication study. J Am Acad Child Adolesc Psychiatry 38, 1230-1236.

46. Canals J, Hernández-Martínez C, Cosi S et al. (2012) Examination of a cutoff score for the Screen for Child Anxiety Related Emotional Disorders (SCARED) in a nonclinical Spanish population. J Anxiety Disord 26, 785-791.

47. Kovacs M (1992) Children's Depression Inventory. North Tonawanda, NY: Multi-Health Systems, Inc.

48. Del Barrio V \& Carrasco MA (2004) CDI. Inventario de depresión infantil. Madrid: TEA Ediciones.

49. Figueras A, Amador-Campos JA, Gómez-Benito J et al. (2010) Psychometric properties of the Children's Depression Inventory in community and clinical sample. Span J Psychol 13, 990-999.

50. Canals J, Martí-Henneberg C, Fernández-Ballart J et al. (1995) A longitudinal study of depression in urban Spanish pubertal population. Eur Child Adolesc Psychiatry 4, 102-111.

51. Gadow K \& Sprafkin J (1999) Youth's Inventory-4 Manual. Stony Brook, NY: Checkmate Plus, Ltd.

52. Garner DM (1998) Inventario de Trastornos de la Conducta Alimentaria (EDI-2)-Manual. Madrid: TEA Ediciones.

53. Hollingshead AB (2011) Four factor index of social status. Yale J Soc 8, 21-52.

54. Hafeman DM \& Schwartz S (2009) Opening the black box: a motivation for the assessment of mediation. Int J Epidemiol 38, 838-845. 
55. De Rufino Rivas PM, Antolín Guerra O, Casuso Ruiz I et al. (2014) Evaluación del riesgo nutricional de los adolescentes escolarizados en Cantabria. Nutr Hosp 29, 652-657.

56. Gutiérrez-Fisac JL, Guallar-Castillón P, León-Muñoz LM et al. (2012) Prevalence of general and abdominal obesity in the adult population of Spain, 2008-2010: the ENRICA study. Obes Rev 13, 388-392.

57. Sánchez-Cruz JJ, Jiménez-Moleón JJ, Fernández-Quesada F et al. (2013) Prevalence of child and youth obesity in Spain in 2012. Rev Esp Cardiol 66, 371-376.

58. Bonaccio M, Bonanni AE, Di Castelnuovo A et al. (2012) Low income is associated with poor adherence to a Mediterranean diet and a higher prevalence of obesity: cross-sectional results from the Moli-sani study. BMJ Open 2, e001685.

59. Rossi M, Negri E, Bosetti C et al. (2008) Mediterranean diet in relation to body mass index and waist-to-hip ratio. Public Health Nutr 11, 214-217.

60. Martínez-González MA, García-Arellano A, Toledo E et al. (2012) A 14-item Mediterranean diet assessment tool and obesity indexes among high-risk subjects: the PREDIMED trial. PLoS One 7, e43134.

61. Schröder H, Marrugat J, Vila J et al. (2004) Adherence to the traditional Mediterranean diet is inversely associated with body mass index and obesity in a Spanish population. J Nutr 134, 3355-3361.

62. Al-Hazzaa HM, Al-Sobayel HI, Abahussain NA et al. (2014) Association of dietary habits with levels of physical activity and screen time among adolescents living in Saudi Arabia. J Hum Nutr Diet 27, 204-213.

63. Leech RM, McNaughton SA \& Timperio A (2014) The clustering of diet, physical activity and sedentary behavior in children and adolescents: a review. Int J Behav Nutr Phys Act 11, 4.

64. Akbaraly TN, Brunner EJ, Ferrie JE et al. (2009) Dietary pattern and depressive symptoms in middle age. Br J Psychiatry 195, 408-413.

65. Jacka FN, Rothon C, Taylor S et al. (2013) Diet quality and mental health problems in adolescents from East London: a prospective study. Soc Psychiatry Psychiatr Epidemiol 48, 1297-1306.

66. Rienks J, Dobson AJ \& Mishra GD (2013) Mediterranean dietary pattern and prevalence and incidence of depressive symptoms in mid-aged women: results from a large community-based prospective study. Eur J Clin Nutr 67, 75-82.
67. Kingsbury M, Dupuis G, Jacka F et al. (2016) Associations between fruit and vegetable consumption and depressive symptoms: evidence from a national Canadian longitudinal survey. J Epidemiol Community Health 70, 155-161.

68. O'Neil A, Quirk SE, Housden S et al. (2014) Relationship between diet and mental health in children and adolescents: a systematic review. Am J Public Health 104, e31-e42.

69. Bottiglieri $\mathrm{T}$ (1996) Folate, vitamin $\mathrm{B}_{12}$, and neuropsychiatric disorders. Nutr Rev 54, 382-390.

70. Esposito K, Marfella R, Ciotola M et al. (2004) Effect of a Mediterranean-style diet on endothelial dysfunction and markers of vascular inflammation in the metabolic syndrome: a randomized trial. JAMA 292, 1440-1446.

71. Karatzi K, Papamichael C, Karatzis E et al. (2008) Postprandial improvement of endothelial function by red wine and olive oil antioxidants: a synergistic effect of components of the Mediterranean diet. J Am Coll Nutr 27, 448-453.

72. Mena MP, Sacanella E, Vázquez-Agell M et al. (2009) Inhibition of circulating immune cell activation: a molecular antiinflammatory effect of the Mediterranean diet. Am J Clin Nutr 89, 248-256.

73. Salas-Salvadó J, Fernández-Ballart J, Ros E et al. (2008) Effect of a Mediterranean diet supplemented with nuts on metabolic syndrome status: one-year results of the PREDIMED randomized trial. Arch Intern Med 168, 2449-2458.

74. Fulkerson JA, Sherwood NE, Perry CL et al. (2004) Depressive symptoms and adolescent eating and health behaviors: a multifaceted view in a population-based sample. Prev Med 38, 865-875.

75. Jacka FN, Kremer PJ, Berk M et al. (2011) A prospective study of diet quality and mental health in adolescents. PLOS One 6, e24805.

76. Mooreville M, Shomaker LB, Reina SA et al. (2014) Depressive symptoms and observed eating in youth. Appetite 75, 141-149.

77. Wurtman RJ \& Wurtman JJ (1995) Brain serotonin, carbohydrate-craving, obesity and depression. Obes Res $\mathbf{3}$, Suppl. 4, 477S-480S.

78. Sotos-Prieto M, Santos-Beneit G, Pocock S et al. (2015) Parental and self-reported dietary and physical activity habits in pre-school children and their socio-economic determinants. Public Health Nutr 18, 275-285. 